\section{Cahiers de littérature orale}

$70 \mid 2011$

L'adresse indirecte ou la parole détournée

\title{
Minna SKAFTE JENSEN, Writing Homer: a study based on results from modern fieldwork
}

Manon Brouillet

\section{OpenEdition}

Journals

Édition électronique

URL : https://journals.openedition.org/clo/1373

DOI : $10.4000 /$ clo. 1373

ISSN : 2266-1816

Éditeur

INALCO

Édition imprimée

Date de publication : 30 décembre 2011

Pagination : 177-181

ISBN : 978-2-85831-202-3

ISSN : 0396-891X

\section{Référence électronique}

Manon Brouillet, « Minna SkAFTE Jensen, Writing Homer: a study based on results from modern fieldwork », Cahiers de littérature orale [En ligne], 70 | 2011, mis en ligne le 18 mars 2013, consulté le 01 juillet 2021. URL : http://journals.openedition.org/clo/1373 ; DOI : https://doi.org/10.4000/clo.1373

Ce document a été généré automatiquement le 1 juillet 2021.

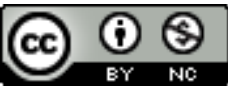

Cahiers de littérature orale est mis à disposition selon les termes de la Licence Creative Commons Attribution - Pas d'Utilisation Commerciale 4.0 International. 


\title{
Minna SKAFTE JENSEN, Writing Homer: a study based on results from modern fieldwork
}

\author{
Manon Brouillet
}

\section{RÉFÉRENCE}

Minna SKAFTE JENSEN, Writing Homer: a study based on results from modern fieldwork, Scientia Danica, Series H, Humanistica 8, 4, Copenhagen, Det Kongelige Danske Videnskabernes Selskab/The Royal Danish Academy of Sciences and Letters, 2011, 439 p. ISBN : 978-87-7304-361-5.

1 L'auteur s'attaque dans cet ouvrage à une des questions les plus débattues par les antiquisants : la question homérique, c'est-à-dire la question de la création, de la composition et de la mise par écrit de l'Iliade et de l'odyssée, ainsi que celle de l'identité de celui qu'on appelle Homère. L'étude se veut un dialogue avec les grandes thèses soutenues aujourd'hui, en particulier celle de Martin West (selon qui un poète a composé et mis par écrit les épopées et a ensuite corrigé son « manuscrit » tout au long de sa vie) et celle de Gregory Nagy (qui a construit un modèle évolutionniste avec une fixation progressive et différentes étapes dans la mise par écrit dont les variantes seraient les traces). Mais c'est aussi une réponse à celui qui a révolutionné la question homérique au début du siècle dernier : Milman Parry, dont les thèses sont longuement évoquées, tout comme celles de son disciple Albert Lord. Minna Skafte Jensen est en effet l'héritière directe de Parry. Il y a près d'un siècle, Parry a montré que l'Iliade et l'Odyssée présentaient les caractéristiques d'une composition orale grâce, notamment, à l'observation des épopées serbo-croates composées oralement. Une fois les conclusions de Parry acceptées, et elles l'ont largement été, les homéristes se sont retrouvés devant un nouveau problème : comment rendre compte de ces textes, composés oralement, mais qui se présentent pour nous sous la forme écrite ? Ainsi, la question cruciale de cet 
ouvrage est celle du rapport entre l'oral et l'écrit, question qui ne concerne pas seulement les hellénistes et à laquelle ils ne peuvent répondre seuls. À l'inverse, toute tentative de réponse appliquée à l'épopée homérique peut être utile à ceux qui s'intéressent à ce problème. L'enjeu est général et pluridisciplinaire et la démarche de M. Jensen dépasse largement le cadre des études homériques.

2 L'ouvrage s'articule essentiellement autour d'une démarche comparatiste. Son but est de s'appuyer sur les traditions épiques vivantes, qui sont désormais documentées avec précision, afin de comprendre comment les épopées homériques ont pu être composées et surtout mises par écrit. Avec méthode, l'auteur s'attache d'abord (chapitre I) à sélectionner les épopées qui seront les plus pertinentes. Celles-ci sont au nombre de six, deux africaines, trois indiennes et une d'Asie centrale. M. Jensen présente ces épopées, leurs thèmes, leurs caractéristiques stylistiques et surtout la manière dont elles ont été étudiées (par un ou plusieurs ethnologues, par l'intermédiaire d'un ou plusieurs chanteurs, avec un enregistrement, une mise par écrit sous la dictée...). Le choix de M. Jensen a été guidé par des critères stylistiques (il s'agit d'épopées, le plus souvent sous forme poétique), mais surtout parce qu'elles avaient été décrites de manière intéressante par rapport à la théorie de l'oralité. Elle reprend ensuite (chapitre II) la théorie de l'oralité développée par Lord et Parry et cherche à l'appliquer aux épopées qu'elle a sélectionnées. Si les motifs les plus précis (comme l'épithète formulaire) ne sont pas transposables, les lois de l'épopée orale définies par les deux savants sont assez stables pour supporter la généralisation. Or, une de ces lois veut que la composition ait lieu au moment même de la performance, ce qui établit de fait une rupture avec la littérature écrite. Au chapitre III, en développant différents exemples des épopées sélectionnées, $M$. Jensen montre l'influence du contexte de la performance sur le contenu de l'épopée. Celle-ci a pour conséquence la constante variation entre les performances (chapitre IV). Décisive est l'introduction de la notion de «texte mental ", expression que l'auteur reprend à Lauri Honko, spécialiste de l'épopée de Siri (Sudouest de l'Inde). C'est ce phénomène que l'auteur cherche à définir avec précision afin de comprendre le passage d'une composition orale à un texte écrit. Chaque chanteur modifie sans cesse son texte mental : chaque performance doit en effet correspondre à la situation unique dans laquelle elle a lieu. On remarque la difficulté bien naturelle qu'a Jensen à se départir des pratiques de l'écrit, notamment par son utilisation des termes « text » et « editing » qui soulignent d'une certaine manière la proximité avec la pratique de l'écrit bien plus qu'ils ne laissent voir une différence. L'auteur laisse de côté pour un moment (chapitre v) les épopées d'aujourd'hui pour s'intéresser aux témoignages antiques, internes et externes à l'épopée, qui permettent de rendre compte des conditions dans lesquelles se déroulait une performance épique dans l'Antiquité. C'est aussi le moment pour elle de rappeler la distinction entre les deux usages du nom propre Homère : il désigne d'abord celui qui aurait composé, de quelque manière que ce soit, l'Iliade et l'Odyssée, mais aussi toute la tradition autour de la guerre de Troie. Elle montre de manière convaincante que jusqu'à Platon, les sources iconographiques et littéraires qui mentionnent le cycle troyen ne font jamais allusion à l'Iliade et l'Odyssée telles que nous les connaissons aujourd'hui (de même leurs noms ne sont pas mentionnés dans les textes tandis que, dès Hérodote, apparaît le nom d'Homère). $M$. Jensen aborde ensuite deux concepts développés par les homéristes : celui de textes de transition (Albert Lord, chapitre vI) et celui de fixation graduelle (Gregory Nagy, chapitre VII). Pour Lord, il existe des phases de transition de l'oral à 
l'écrit, mais pas de texte de transition, car les deux techniques s'excluent l'une l'autre. Le texte de transition combinerait les deux techniques: composé à l'écrit, il ferait l'objet d'une performance orale ou, composé oralement, il donnerait lieu à une œuvre écrite. Aucune de ces pratiques n'a jamais été attestée sur le terrain par des anthropologues. Même lettrés, les poètes d'aujourd'hui n'utilisent pas l'écrit comme support de leur performance. Nagy s'élève, lui, contre l'idée qu'il existerait un seul et unique "génie homérique » à l'origine des poèmes que nous connaissons. Pour lui, ces épopées sont le résultat d'une cristallisation progressive: alors que le système de composition est toujours le même, les textes varient et la fixation est le résultat d'un processus séculaire où la prolifération des performances conduit à la standardisation des épopées, standardisation facilitée par l'usage de l'écrit. La principale objection de M. Jensen à G. Nagy est que nous n'avons pas de variantes de l'Iliade et de l'Odyssée. Nous ne connaissons que des variations mineures; il n'y a donc pu avoir qu'une seule mise par écrit, à un moment précis. D'autre part, une écriture graduelle n'aurait pas produit des intrigues aussi cohérentes que celles que nous connaissons. Une fois ces épopées mises par écrit, la tradition a continué à exister, des performances ont eu lieu durant toute l'Antiquité, mais sans être affectées par l'existence de ces épopées et sans plus avoir d'influence sur elles (chapitre viII). Si l'Iliade et l'Odyssée ont connu le succès que nous savons, ce n'est pas parce que les chanteurs les ont chantées dans tout le monde grec, mais parce que, étant les premières épopées rédigées, elles ont été utilisées dans les écoles et en sont venues à former le socle commun d'éducation dans le monde hellénophone. Le chapitre Ix est le lieu d'un retour au comparatisme et sans doute sa mise en application la plus intéressante. L'auteur étudie comment et pourquoi les épopées modernes ont été enregistrées par écrit et cherche à appliquer ces modèles aux deux épopées homériques. M. Jensen constate que ni le public ni le chanteur ne ressentent jamais le besoin d'enregistrer une épopée, mais que cela est toujours fait à la demande d'un tiers. Son hypothèse est que l'Iliade puis l'odyssée ont été dictées à un scribe par un chanteur à l'occasion de sa victoire aux grandes Panathénées durant la tyrannie des Pisistratides, en 522, sans doute à la demande du tyran. Ce qui est considéré comme des interpolations et ajouts "athéniens" serait en fait des émendations faites par le poète lui-même à son texte mental pour satisfaire un public athénien. De même, la division en vingt-quatre chants (chapitre $\mathrm{x}$ ) paraît correspondre à un enregistrement typique qui aurait pu se dérouler sur vingt-quatre jours, avec des transitions tout à fait représentatives d'une reprise après un arrêt dans l'enregistrement. L'hypothèse d'une fixation de l'Iliade et de l'odyssée à l'occasion des Panathénées n'est pas nouvelle, mais l'originalité de M. Jensen est d'affirmer que les épopées n'ont pas seulement été fixées à ce moment-là, après une longue existence orale et par des rédacteurs athéniens différents des compositeurs primitifs, mais qu'elles ont bel et bien été composées à cette occasion et aussitôt mises par écrit de manière définitive.

3 Jensen livre un ouvrage très clair et rendu aisément maniable par de courts résumés à la fin de chaque chapitre. Sa thèse, celle d'un enregistrement unique, est convaincante quand elle s'appuie avec précision sur une démarche comparatiste, ce qui est le cas dans toute la première partie de l'ouvrage. En revanche, quand elle cherche à dater et contextualiser précisément cet enregistrement, les arguments se font selon nous moins probants (ainsi le chapitre XI qui interprète trois statuettes trouvées à Athènes comme des témoignages de l'enregistrement pisistratide de l'Iliade et de l'Odyssée). Pour donner à la notion de performance toute l'importance qu'elle mérite, l'auteur fait un usage très 
appréciable du comparatisme. Elle présente précisément les différents objets comparés et surtout justifie toujours pourquoi ces objets sont comparables. Sa démarche est construite selon un mouvement de balancier: elle montre d'abord que l'on peut appliquer les concepts créés par des homéristes à des épopées orales actuelles puis déduit des épopées actuelles les conditions de mise par écrit de l'Iliade et de l'Odyssée. C'est une thèse forte que Minna Skafte Jensen s'emploie à démontrer par différents moyens (comparatisme, mais aussi étude interne du texte, sources littéraires et iconographiques antiques) dont on peut ou non être convaincu, mais dans tous les cas son appropriation des travaux des anthropologues est un bon exemple de l'usage qui peut être fait du comparatisme par les antiquisants. 